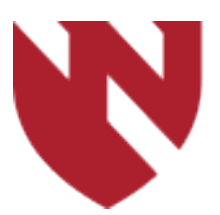

December 2019

\title{
A Clinical and Cost Effectiveness Comparison of Traditional VNS Therapy to Aspire Sr (106)
}

\author{
Krishna Galla \\ University of Nebraska Medical Center \\ Kalyan Sajja \\ University of Nebraska Medical Center \\ Christopher Wichman \\ University of Nebraska Medical Center \\ Hongmei Wang \\ University of Nebraska Medical Center \\ Deepak Madhavan \\ University of Nebraska Medical Center
}

Tell us how you used this information in this short survey.

Follow this and additional works at: https://digitalcommons.unmc.edu/gmerj

Part of the Higher Education Commons, and the Medicine and Health Sciences Commons

\section{Recommended Citation}

Galla, K., Sajja, K., Wichman, C., Wang, H., , Madhavan, D. A Clinical and Cost Effectiveness Comparison of Traditional VNS Therapy to Aspire Sr (106). Graduate Medical Education Research Journal. 2019 Dec 13; 1(1). https://digitalcommons.unmc.edu/gmerj/vol1/iss1/50 


\section{A Clinical and Cost Effectiveness Comparison of Traditional VNS Therapy to Aspire $\mathrm{Sr}$ (106)}

\section{Creative Commons License}

\section{(c) (1) $\Theta(9$}

This work is licensed under a Creative Commons Attribution-Noncommercial-No Derivative Works 4.0 License. 
$>0.05$, ANOVA). Postmortem evaluation revealed that 2-3 hepatic veins were transected by injury type 1, 1-2 portal vein branches plus 1 hepatic vein were transected by injury type 3 (LLL transection).

Conclusions: The hemorrhage severity of the central stellate injury was inadequate, while hemorrhage from excision of a proximal branch of the portal vein appeared to be too severe (i.e., the subjects died too quickly).
Near-transection of the hepatic LLL at its base appeared to yield hemorrhage of appropriate severity, as indicated by the $40 \% 1$-hour mortality and intermediate values for MAP, blood loss, and other variables. Our plan is to use this latter injury model in the development of therapies for noncompressible truncal hemorrhage.

https://doi.org/10.32873/unmc.dc.gmerj.1.1.047

\section{Exogenous Testosterone Supplementation as Cause of Ischemic Stroke, Mediated through Secondary Erythrocytosis \\ Fuad-al Ali, Amy Hellman, Pierre Fayad}

Mentor: Pierre Fayad

Program: Neurology

Background: The use of exogenous testosterone has become more prevalent in recent years but the effects of such use in regards to potential cerebrovascular disease have yet to come to a conclusion. In this case report, we discuss a patient who suffered an ischemic stroke due to testosterone supplementation.

Case presentation: A middle-aged patient with history of hypertension and hyperlipidemia that presented with falls, difficulty performing simple tasks and slurred speech. Patient had been taking testosterone injections every 6 months for the prior 2 years for loss of energy and reported low levels. Lab work revealed a hemoglobin of $20 \mathrm{~g} / \mathrm{dL}$ and a hematocrit of $51.5 \%$. MRI of the brain showed a left striatocapsular ischemic infarct. He was given the diagnosis of ischemic stroke due to polycythemia and thrombus formation secondary to testosterone supplementation.

Discussion: Testosterone and dihydrotestosterone (DHT) has both protective and adverse effects. Both low and high levels of testosterone and DHT have an association with ischemic stroke and there may be an optimal level that is safe.

Conclusion: The risk of ischemic stroke may be related to testosterone and DHT levels but no clear guidelines exist on optimal levels in supplementation. Thus, clinicians should be aware of the potential risk and monitor androgen and hematocrit levels.

https://doi.org/10.32873/unmc.dc.gmerj.1.1.048

\section{Pharmacological Treatment of Status Epilepticus at UNMC: Assessment of Seizure Outcomes} Hae Young Baang, Nicholas Swingle, Kalyan Sajja, Deepak Madhavan, Olga Taraschenko

Mentor: Olga Taraschenko

Program: Neurology

Objective: To assess the effectiveness of treatment for status epilepticus (SE) at UNMC and determine patient health outcomes related to the delayed control of seizures

Design/Methods: Retrospective chart review was performed on 1565 adult patients who underwent continuous video electroencephalography (cvEEG) for suspected seizures between January 1, 2012 and February 28, 2018.

Results: 49 patients had and were treated for 64 incidences of convulsive or nonconvulsive
SE recorded on cvEEG or clinical seizures longer than 5 min which were documented by a neurologist. Among 64 cases, 55 incidences required the second AED treatment, and 40 out of 55 cases were treated with the third AED subsequently. The mean seizure detection-to-needle time for the first, second, and third antiepileptic drugs were 167, 239, and 297 minutes, respectively. Benzodiazepines were administered as the first AED in only $46 \%$ of all treated cases. The duration of the hospital stays in patients treated with the first AED within 60 min after seizure detection was shorter compared to those whose treatment was delayed (23.0 vs.
33.6 days, $p=0.41$ ). The mortality rate in the former group was lower than that in the group which did not receive AEDs within $60 \mathrm{~min}$ (28.6 vs. $53.6 \%, \mathrm{p}=0.02$ ).

Conclusion: The current treatment approaches for SE at UNMC are inefficient with significant delay in delivery of medications after seizure detection; they may contribute to the prolonged hospital stay and increased mortality. In order to improve patient care, the existing protocol for the treatment of SE at UNMC must be revised.

https://doi.org/10.32873/unmc.dc.gmerj.1.1.049

\section{A Clinical and Cost Effectiveness Comparison of Traditional VNS Therapy to Aspire Sr (106)}

Krishna Mourya Galla, Kalyan Sajja, Christopher Wichman, Hongmei Wang, Deepak Madhavan

Mentor: Deepak Madhavan

Program: Neurology

Background: VNS Therapy has demonstrated overall cost savings in patients with drug- resistant epilepsy. The overall goal of this project is to evaluate the clinical and cost effectiveness of the Aspire SR 106 VNS (Vagus Nerve Stimulator) system, using retrospective patient data. We anticipate the extra dimension of closed-loop autostimulation in the Aspire 106 model will demonstrate more efficient utilization of health care resources. 
Methods: Data were collected using EPIC ${ }^{\circledR}$ starting 12 months prior to VNS replacement to Aspire ${ }^{\circledR}$ device to 12 months after implantation date in 3-month blocks. Seizure frequency before and after the VNS implantation as well as medication changes were identified from review of clinic notes. Cost was estimated based on the number of visits for ER, clinic, hospital, and ICU using hospital average cost data. As cost distributions are skewed to the right, we also calculated log total costs for the trend analysis using segmented model. For data analysis segmented Poisson regression was used to establish a trend in usage per quarter.
Results: The trend in Clinic usage was statistically flat $(p>0.05)$ in the preplacement period while the trend in the post placement was downward with an estimate that for every quarter the natural log of the average number of clinic visits decreases by 0.29 . By one-year post placement, the average number of clinic visits had achieved the same level as the pre-placement period. The trends in ER and hospital usage were flat in both the pre and post placement periods. The median number of CPS in the year after placement of the Aspire 106 VNS was statistically smaller by $1(\mathrm{p}=0.0161)$ CPS than the median number of CPS in the year prior to placement.
Conclusion: Analysis of the above data yielded two main findings: there were no demonstrable cost savings within a 12-month period following VNS implantation, but the median burden of Complex Partial Seizures (CPS) within our cohort revealed a 50\% decline, which was statistically significant. However, overall costs post-implantation showed a consistent downward trajectory, with approximation to pre-implantation costs within the 12-month timeframe.

https://doi.org/10.32873/unmc.dc.gmerj.1.1.050

thalamus-midbrain junction just posterior to the red nucleus.

Conclusion: This case illustrates how a punctate single unilateral lesion can present with vertical supranuclear gaze palsy and horizontal eye movement abnormalities.

https://doi.org/10.32873/unmc.dc.gmerj.1.1.051
Method: Characterize the eye movement deficits in this patient and correlate the MRI diffusion restriction findings.
Results: This patient presented with vertical gaze palsy and impaired adduction of the left eye. Impaired smooth pursuits up and down clinically localized to damage in the interstitial nucleus of Cajal. Impaired saccades up and down localized to the rostral interstitial nuclei of the medial longitudinal fasciculus. MRI showed diffusion restriction in the left

\section{Paroxysmal Autonomic Instability and Dystonia Following a Motor Vehicle Accident Matthew Purbaugh, Krishna Galla, Marco A. Gonzalez Castellon}

Mentor: Marco A. Gonzalez Castellon

Program: Neurology

Background: We were consulted a young adult patient who presented following a motor vehicle accident. The patient presented with diffuse axonal injury, hemorrhage of left basal ganglia increased intra-cranial pressure and other injuries. The patient was intubated, an intraventricular shunt had been removed and a 7-day course of Keppra prophylaxis had been finished. The patient was stable until the morning we were consulted.

Methods: On exam the patient had episodes of extensor posturing, diffuse dystonia and high frequency, low amplitude tremors, with autonomic instability limited to these spells.
The patient was found to have $3+$ reflexes throughout. Brain stem reflexes were intact. Stimuli caused extensor posturing, rigidity and autonomic instability. Continuous VEEG showed epileptiform activity that did not correlate with spells on video. Lab was non-specific expect for a reelevated CK. We loaded Keppra and prn diazepam. This failed to control the symptoms.

Results: We arrived at a diagnosis of paroxysmal autonomic instability and dystonia (PAID) syndrome. Treatment with clonazepam, Gabapentin, baclofen and propranolol stabilized the autonomic system and resolved the dystonia. The CK began to drop and the patient began to respond to commands.
Conclusions: PAID most commonly affects young persons following severe TBI. Potentially caused by disinhibition of sympathoexcitatory regions leading to cortically provoked catecholamine surges causing autonomic instability. Dystonia is secondary to disruption of the pontine and vestibular nuclei. PAID should on the differential diagnosis of TBI patients who are not responding to typical treatments. We had success treating with Clonazepam, gabapentin, baclofen and propranolol. https://doi.org/10.32873/unmc.dc.gmerj.1.1.052

\section{Diagnostic Challenges in The Radiographic Distinction Between Demyelinating Lesions and Primary Brain Neoplasms \\ Erin L. Smith and Geetanjali Rathore}

Mentor: Geetanjali Rathore

Program: Neurology

Introduction: The differentiation between demyelinating lesions and primary brain neoplasms is difficult based on radiography alone, resulting in misdiagnoses and delay in treatment. Here we present two cases with MRI abnormalities suggestive of either demyelination or neoplasm. Case 1 was treated with steroids for a demyelinating process, but was found on biopsy to have an anaplastic astrocytoma that was unresectable by the time of diagnosis. Case 2 was thought to be glioma but was later determined to be tumefactive demyelination. In both cases, there were delays due to the limitations of MR imaging.
Methods: The cases of the two patients were reviewed via EMR. A literature review investigated the most sensitive and specific non-invasive diagnostic modalities for diagnosing demyelinating lesions or primary brain neoplasms.

Results: Review of recent literature shows that conventional MR sequences have 